Pacific Journal of Mathematic 


\section{UNIQUENESS OF THE GRAPH OF A RANK THREE GROUP}

DAVID WALES

Let $G$ be a transitive permutation group on a set $\Omega$. Let $\{a\}$ be an element of $\Omega$ and $\Delta(a)$ an orbit of the stabilizer $G_{a}$ of $\{a\}$ in $G$. A graph on the points of $\Omega$ can be constructed by joining $\{a\}$ to $\Delta(a)$ and joining each image of $\{a\}$ by an element of $G$ to the corresponding image of $\Delta(a)$. The permutation group $G$ is said to be of rank 3 if $G_{a}$ has three orbits including $\{a\}$. In this paper, conditions are discussed which ensure that the graph of a rank three permutation group is unique in the following sense. Suppose $G$ is a rank three permutation group and $G_{a}$ has orbits $\{a\}, \Delta(a)$, and $\Gamma(a)$. Is the graph uniquely determined once the permutation representation of $G_{a}$ on $\Delta(a)$ and on $\Gamma(a)$ is known? Conditions involving orbit lengths of $G_{a}$ acting on $\Delta(a)$ and on $\Gamma(a)$ are found which ensure the graph is uniquely determined. The conditions specify how the graph is to be drawn. They give, therefore, a prescription for constructing rank three groups. They shed no light however on the difficult problem of the transitivity of the group of automorphisms of the graph.

A few applications are given at the end to illustrate the method. They are not intended to be exhaustive. The same ideas can be used to show the nonexistence of certain rank three extensions.

2. Construction of the graph from the orbits. We assume $G$ is a transitive rank three permutation group on a set $\Omega$ containing $n$ points. The notation is the same as in [2]. For $\{a\}$ in $\Omega$ let the orbits of $G_{a}$ be $\Delta(a)$ and $\Gamma(a)$. We assume $(\Delta(a))^{g}=\Delta\left(a^{g}\right)$ for all elements $g$ in $G$. A graph is defined by joining $\{a\}$ to the points of $L(a)$ for each $\{a\}$ in $\Omega$. The group $G$ is then a subgroup of the full automorphism group of the graph. We assume that the permutation representation is primitive and that $G$ has even order. We set $|\Delta(a)|=k$, $|\Gamma(a)|=l$.

We now give a description of the graph derived from a consideration of the orbits $\{a\}, \Delta(a)$, and $\Gamma(a)$. Here $\{a\}$ is a specific point in $\Omega$. By definition, $\{a\}$ is joined to each of the points in $A(a)$. Let $\{b\}$ be a point in $\Delta(a)$ and $\{c\}$ a point in $\Gamma(\alpha)$. We will describe the points which are joined to $\{b\}$ and $\{c\}$ in terms of unions of orbits of $G_{a b}$ and $G_{a c}$. Here $G_{a b}$ is the stabilizer of $\{a\}$ and $\{b\}$ and $G_{a c}$ is the stabilizer of $\{a\}$ and $\{c\}$.

The parameters $\lambda$ and $\mu$ are integers defined as follows, [2, §2]. 
If $b \in \Delta(a),|\Delta(a) \cap \Delta(b)|=\lambda$ and if $c \in \Gamma(a),|\Delta(a) \cap \Delta(c)|=\mu$. These values are independent of the particular element $\{b\}$ chosen in $\Delta(a)$ and of the particular element $\{c\}$ chosen in $\Gamma(\alpha)$. As $G$ has even order, the orbits are self paired by $[4,16.5]$. This means that $\{b\}$ is joined to $\{a\}$ and that $\{c\}$ is not. As $G$ is primitive $\mu \neq 0$ [2]. In particular then $\lambda \neq k-1$ by [2, lemma 5$]$.

The point $\{b\}$ is joined to the $k$ points of $\Delta(b)$. There are $\lambda$ of these points $\Delta(b) \cap \Delta(a)$ in $\Delta(a)$ and so $k-\lambda$ outside $\Delta(a)$. As $\{b\}$ is joined to $\{a\}, k-\lambda-1$ of these points are in $\Gamma(a)$. These points are of course $\Gamma(a) \cap \Delta(b)$. The point $\{c\}$ is joined to the $\mu$ points $\Delta(c) \cap \Delta(a)$ in $\Delta(a)$ and to the $k-\mu$ points $\Delta(c) \cap \Gamma(a)$ in $\Gamma(a)$. We have the following table.

TABLE 1. Points connected to $b$.

location

$$
\{a\}
$$

$\Delta(a)$

$\Gamma(a)$ points

$a$
$\Delta(a) \cap \Delta(b)$
$\Gamma(a) \cap \Delta(b)$

number

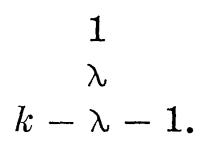

Points connected to $c$.

$\begin{array}{cccc} & \text { location } & \text { points } & \text { number } \\ \text { (iv) } & \Delta(a) & \Delta(a) \cap \Delta(c) & \mu \\ \text { (v) } & \Gamma(a) & \Gamma(a) \cap \Delta(c) & k-\mu .\end{array}$

Once the subsets in the various categories are found for all points $b \in \Delta(a)$ and $c \in \Gamma(a)$ the graph can be completely drawn.

The points in category (iii) or (iv) determine all lines joining points in $\Delta(a)$ to points in $\Gamma(a)$. These lines can be determined once the sets in (iii) are known for each $b \in \Delta(a)$ or once the sets in (iv) are known for each $c$ in $\Gamma(a)$. Counting these lines each way gives again [2, Lemma 5] $\mu l=k(k-\lambda-1)$. The graph can be drawn once the sets in category (ii), (v) and either (iii) or (iv) are determined for every $b$ in $\Delta(a)$ and $c$ in $\Gamma(a)$.

It is clear that $G_{a b}$ leaves invariant $\Delta(a), \Gamma(a)$, and $\Delta(b)$. Therefore $\Delta(a) \cap \Delta(b)$ and $\Gamma(a) \cap \Delta(b)$ are invariant under $G_{a b}$. It follows that these two sets are unions of orbits of $G_{a b}$. Similarly, the sets $\Delta(a) \cap \Delta(c)$ and $\Gamma(a) \cap \Delta(c)$ are unions of orbits of $G_{a c}$. If the unions of orbits in the sets in category (ii), (v), and either of (iii) or (iv) can be chosen uniquely, the graph can be drawn in only one way.

We know that $\Delta(a) \cap \Delta(b)$ is invariant under $G_{a b}$. Also $\Delta(a) \cap \Gamma(b)=$ $\Delta(a)-\{a\}-\Delta(b)$ is invariant under $G_{a b}$. Also $|\Delta(a) \cap \Gamma(b)|=k-\lambda-1$. There are now two sets of length $k-\lambda-1, \Delta(a) \cap \Gamma(b)$ and $\Gamma(a) \cap \Delta(b)$ invariant under $G_{a b}$. We will show they are permutation isomorphic. 
This will provide a further restriction on the choice of either $\Delta(a) \cap \Delta(b)$ or $\Gamma(a) \cap \Delta(b)$. Similarly $\Delta(a) \cap \Gamma(c)$ and $\Gamma(a) \cap \Delta(c)$ are permutation isomorphic $G_{a c}$ invariant subsets.

It follows from $[4,16.4]$ that there is an element $\tau$ in $G$ which interchanges $\{a\}$ and $\{b\}$ and an element $\tau_{1}$ in $G$ which interchanges $\{a\}$ and $\{c\}$. Here the orbits are self paired as $G$ has even order. Clearly, $\tau$ normalizes $G_{a b}$ and $\tau_{1}$ normalizes $G_{a c}$. We will show that $\tau$ maps $\Delta(a) \cap \Gamma(b)$ to $\Gamma(a) \cap \Delta(b)$ and that $\tau_{1}$ maps $\Delta(a) \cap \Gamma(c)$ to $\Gamma(a) \cap \Delta(c)$. If $X \in \Delta(a) \cap \Gamma(b)$ and $g \in G_{a b}$, then $(X)^{g \tau}=\left(X^{\tau}\right)^{\tau^{-1} g \tau}$. This means that the map of $\Delta(a) \cap \Gamma(b)$ to $\Gamma(a) \cap \Delta(b)$ by $\tau$ together with the inner automorphism of $G_{a b}$ to itself given by $\tau$ provides a permutation isomorphism between the action of $G_{a b}$ on $\Delta(a) \cap \Gamma(b)$ and an action of $G_{a b}$ on $\Gamma(a) \cap \Delta(b)$. Similarly, $\tau_{1}$ provides a permutation isomorphism between the action of $G_{a c}$ on $\Delta(a) \cap \Gamma(c)$ and an action of $G_{a c}$ on $\Gamma(a) \cap \Delta(c)$.

We show now that $\tau$ maps $\Delta(a) \cap \Gamma(b)$ to $\Gamma(a) \cap \Delta(b)$ and $\tau_{1}$ maps $\Delta(a) \cap \Gamma(c)$ to $\Gamma(a) \cap \Delta(c)$. Now $a^{\tau}=b$ and $b^{\tau}=a$ and so $(\Delta(a))^{\tau}=$ $\Delta\left(a^{\tau}\right)=\Delta(b), \quad(\Gamma(b))^{\tau}=\Gamma\left(b^{\tau}\right)=\Gamma(a)$. This means $\quad(\Delta(a) \cap \Gamma(b))^{\tau}=$ $(\Lambda(a))^{\tau} \cap(\Gamma(b))^{\tau}=\Delta(b) \cap \Gamma(a)$. Similarly as $a^{\tau_{1}}=c$ and $c^{\tau_{1}}=a$ we have $(\Delta(a) \cap \Gamma(c))^{\tau_{1}}=\Gamma(a) \cap \Delta(c)$. We now have proved the following theorem.

THEOREM 1. Let $G$ be a primitive rank three permutation group of even order. Suppose $\{a\}, \Delta(a), \Gamma(a)$ are the orbits of $G_{a}$ and the action of $G_{a}$ on these orbits is known. As well, assume that the parameters $\lambda$ and $\mu$ are determined. Let $\{b\}$ be an element in $\Delta(a)$ and $\{c\}$ an element in $\Gamma(a)$. Then the following union of orbits of $G_{a b}$ and $G_{a c}$ must occur.

TABLE 2. Union of Orbits of $G_{a b}$.

location

$$
\Delta(a)
$$

$\Delta(a)$

$\Gamma(a)$

$\Gamma(a)$ points

$\Delta(a) \cap \Delta(b)$

$\Delta(a) \cap \Gamma(b)$

$\Gamma(a) \cap \Delta(b)$

$\Gamma(a) \cap \Gamma(b)$ number of points

$$
\begin{gathered}
\lambda \\
k-\lambda-1 \\
k-\lambda-1 \\
l-k+\lambda+1 .
\end{gathered}
$$

Union of Orbits of $G_{a c}$.

(viii) location

$\Delta(a)$

$\Delta(a)$

$\Gamma(a)$

$\Gamma(a)$ points

$\Delta(a) \cap \Delta(c)$

$\Delta(a) \cap \Gamma(c)$

$\Gamma(a) \cap \Delta(c)$

$\Gamma(a) \cap \Gamma(c)$ number

$$
\begin{gathered}
\mu \\
k-\mu \\
k-\mu \\
l-k+\mu .
\end{gathered}
$$


The graph can be drawn once the sets in (i), (vii) and either of (iii) or $(\mathrm{v})$ are determined. The permutation representations of $G_{a b}$ on $\Delta(a) \cap \Gamma(b)$ and $\Gamma(a) \cap \Delta(b)$ are permutation isomorphic as are the permutation representations of $G_{a c}$ on $\Delta(a) \cap \Gamma(c)$ and on $\Gamma^{\prime}(a) \cap \Delta(c)$. When these unions of orbits can be chosen in only one way the graph can be given uniquely.

REMARK 1. Suppose $G_{a c}$ acting on $\Delta(a)$ has a unique union of orbits of length $\mu$ and further the representation of $G_{a}$ on $I^{\prime}(\alpha)$ is primitive. Then this representation of $G_{a}$ on $\Gamma(a)$ is the representation given by the distinct images by $G_{a}$ of these unique $\mu$ points.

REMARK 2. The nonexistence of rank three extensions can quite often be determined by showing there is no union of orbits of the appropriate lengths. We do not however give any examples of this.

3. Applications. We apply Theorem 1 in several cases. We first consider the Higman-Sims group defined in [3]. Here $G_{a} \cong M_{22}$ has orbits of length 1,22 , and 77 . There is only one permutation group of $M_{22}$ on 22 points. It is doubly transitive. This means $\lambda$ must be zero. Using [2, Lemma 5] we see $\mu=6$. In particular, $G_{a b}$ acting on $A(\alpha)$ has a unique orbit of length zero. From the character table of $M_{22}$ it is clear that a transitive permutation representation of degree 77 must be of rank 3 with permutation character degrees 1 , 21 , and 55. There is therefore a unique orbit of $G_{a c}$ acting on $\Gamma(\alpha)$ of length 16. The restriction of a Sylow 2-group of $M_{22}$ to 22 points has a unique sum of orbits of length six. In fact, the orbit lengths are 2, 4, and 16. A Sylow 2-group must be in the stabilizer of a point on 77-letters. It follows that the stabilizer of these 6 points in $M_{22}$ must be the stabilizer of a point on 77-letters and so the permutation representation on these 77-letters is unique. The conditions of Theorem 1 are met and the uniqueness of the graph is shown. The uniqueness of the Higman-Sims group as a rank three extension of $M_{22}$ is now immediate from the definition in [3].

We next consider a rank three extension of $\operatorname{PSL}(2,9)$ on orbits of length 1,10 , and 45 to give PSL $(3,4)$. This permutation representation was first mentioned to the authors knowledge by Grant Mitchel in a Princeton University thesis in 1910 . The group $\operatorname{PSL}(2,9) \cong A_{6}$ has exactly one permutation representation on 10 letters and exactly one on 45 letters. The 10 letters are the cosets of a Sylow 3-normalizer and the 45 letters are the cosets of a Sylow 2-subgroup. These are the projective points over $G F(9)$ and unordered pairs of distinct projective points. The representation on 10 letters is doubly transitive and so $\lambda=0$. It is easy to check with $[2$, Lemma 5] that $\mu=2$. The re- 
striction of a Sylow 2-group to 10 letters has orbits of length 2, 4, and 4. There is a unique orbit of length 2. On each of the orbits of length 4 a Sylow 2-group is faithfully represented. The permutation representation on 45 points is the permutation representation on the images of the orbit of length 2. The orbit lengths of the stabilizer of a point are $1,8,8,2,2,4,4,8,8$. We must associate two orbits of length 4 with this point. This can be done in only one way and so the graph is unique. The uniqueness of $\operatorname{PSL}(3,4)$ as a rank three extension of $\operatorname{PSL}(2,9)$ is now easy to show.

We next consider a rank three extension of $U_{3}(3)$ on orbits of lengths 1, 36, and 63 described in [1]. As shown in [1] the permutation representation on 36 letters is unique. The stabilizer of a point on 36 letters has orbits of length $1,7,7,21$. This means $\lambda=0,7$, $14,21$, or 28 . The only one consistent with [2, Lemmas 6,7$]$ is $\lambda=14$, $\mu=12$. For simplicity we assume the permutation representation on 63 points is the one used in [1] on the cosets of the centralizer of an involution. The stabilizer of a point on 63 points has orbit lengths 12 and 24 on 36 points and so has a unique orbit of length 12 . The stabilizer of a point in the 63 orbit has orbit lengths $1,6,24$, and 32 and so has a unique orbit of length 24 . The graph is therefore unique. The uniqueness of such a rank three extension can now be easily shown.

Finally, we consider a rank 3 extension on 416 points of the group $H J$ defined above. This extension is isomorphic to $G_{2}(4)$. It was first discovered by Suzuki. He then extended $G_{2}(4)$ to obtain a new simple group. At any rate, we consider a transitive extension of $H J$ to 416 points. The subgroups of small index in $H J$ have indices 100,280 , and 315 . The permutation representation of $G_{2}(4)$ on 416 points is not doubly transitive as $415 \nmid|H J|$. The representation restricted to any subgroup isomorphic to $H J$ must have orbits of length 1,100 and 315. This means there is exactly one conjugate class of subgroups isomorphic to $H J$, the stabilizer of a point. The permutation representation is of rank three.

The stabilizer of a point on 100 letters has orbit lengths 1, 36, and 63. Therefore $\lambda=36$ or 63. It is easy to check using [2, Lemma 5] that $\lambda=36, \mu=20$. The stabilizer of a point on 100 letters has a unique orbit of length 36 . For simplicity we assume the stabilizer of a point on 315 letters is the centralizer of an involution as described in $[1, \S 18]$. This group restricted to 100 points has orbit lengths 20 and 80 as shown in $[1, \S 18]$. It can be shown that there is a unique orbit of length 80 on the 315 points. This shows uniqueness of the graph.

Many of the ideas contained in this paper evolved from discussions with M. Hall, Jr. 


\section{BIBLIOGRAPHY}

1. M. Hall and D. Wales, The simple group of order 604800, J. Algebra 9, 417-450.

2. D. G. Higman, Finite permutation groups of rank 3, Math. Zeit. 86 (1964), 145-156.

3. D. G. Higman and C. C. Sims, A simple group of order 44, 352, 000, Math. Zeit. 105 (1968), 110-113.

4. H. Wielandt, Finite permutation groups, Academic Press, New York, 1964.

Received January 10, 1969.

California Institute of TeChnology 


\section{PACIFIC JOURNAL OF MATHEMATICS}

\section{EDITORS}

H. ROYDEN

Stanford University

Stanford, California

Richard Pierce

University of Washington

Seattle, Washington 98105
J. DugundJI

Department of Mathematics

University of Southern California

Los Angeles, California 90007

BASIL GORDON

University of California

Los Angeles, California 90024

\section{ASSOCIATE EDITORS}

E. F. BECKENBACH

B. H. NeUmanN

F. WOLF

K. YOSHIDA

\section{SUPPORTING INSTITUTIONS}

UNIVERSITY OF BRITISH COLUMBIA

CALIFORNIA INSTITUTE OF TECHNOLOGY

UNIVERSITY OF CALIFORNIA

MONTANA STATE UNIVERSITY

UNIVERSITY OF NEVADA

NEW MEXICO STATE UNIVERSITY

OREGON STATE UNIVERSITY

UNIVERSITY OF OREGON

OSAKA UNIVERSITY

UNIVERSITY OF SOUTHERN CALIFORNIA
STANFORD UNIVERSITY

UNIVERSITY OF TOKYO

UNIVERSITY OF UTAH

WASHINGTON STATE UNIVERSITY

UNIVERSITY OF WASHINGTON

$* * \quad * \quad *$
AMERICAN MATHEMATICAL SOCIETY
CHEVRON RESEARCH CORPORATION
TRW SYSTEMS
NAVAL WEAPONS CENTER




\section{Pacific Journal of Mathematics}

Vol. 30, No. $1 \quad$ September, 1969

William Wells Adams, Simultaneous diophantine approximations and cubic irrationals ..................................... 1

Heinz Bauer and Herbert Stanley Bear, Jr., The part metric in convex

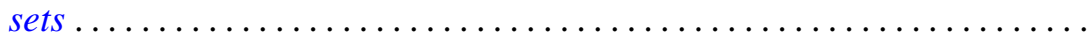

L. Carlitz, A note on exponential sums ...................... 35

Vasily Cateforis, On regular self-injective rings ................. 39

Franz Harpain and Maurice Sion, A representation theorem for measures on infinite dimensional spaces ......................... 47

Richard Earl Hodel, Sum theorems for topological spaces .............. 59

Carl Groos Jockusch, Jr. and Thomas Graham McLaughlin, Countable

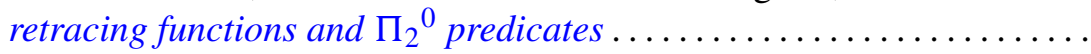

Bjarni Jónsson and George Stephen Monk, Representations of primary

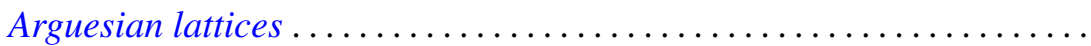

Virginia E. Walsh Knight, A continuous partial order for Peano continua...................................... 141

Kjeld Laursen, Ideal structure in generalized group algebras ........... 155

G. S. Monk, Desargues' law and the representation of primary lattices . . . 175

Hussain Sayid Nur, Singular perturbation of linear partial differential equation with constant coefficients ..........................

Richard Paul Osborne and J. L. Stern, Covering manifolds with cells ... 201

Keith Lowell Phillips and Mitchell Herbert Taibleson, Singular integrals in several variables over a local field...

James Reaves Smith, Local domains with topologically $T$-nilpotent radical....

Donald Platte Squier, Elliptic differential equations with discontinuous coefficients .................................

Tae-il Suh, Algebras formed by the Zorn vector matrix...

Earl J. Taft, Ideals in admissible algebras . .................... 259

Jun Tomiyama, On the tensor products of von Neumann algebras........ 263

David Bertram Wales, Uniqueness of the graph of a rank three group ..... 271

Charles Robert Warner and Robert James Whitley, A characterization of regular maximal ideals ......................... 